\title{
COMMUNAUTÉS ANTIGÉNIQUES DES VENINS ET SYSTÉMATIQUE DES VIPERIDAE
}

\author{
par \\ J. DETRAIT \\ Institut Pasteur, 28 rue du Docteur Roux, 75724 Paris Cedex 15, France
}

$\&$

\section{H. SAINT GIRONS}

Laboratoire d'Evolution des Etres organisés, 105 boulevard Raspail, 75006 Paris, France

\begin{abstract}
A study, by immunoelectrophoresis and immunodiffusion of the shared antigens in venom from 21 species of Viperinae and Crotalinae, representing 10 different genera, has produced a number of results of taxonomic significance which are summarized schematically in the dendrogram shown in fig. 1. For the most part these immunological data confirm the accepted classification of the Viperidae which is based on comparative morphology and biogeography. Some results, on the other hand, such as the isolated position of Atberis, need confirmation with more material. Some of the conclusions reached in this study, however, appear to be of significance for an understanding of the phylogeny of the group. Amongst the Viperinae, the genera Echis and Cerastes are apparently closely related to one another and more similar to the genus Bitis than to the genus Vipera. This latter genus is clearly composed of three groups of species with $V$. russelli occupying an isolated position. Amongst the Crotalinae, the genus Agkistrodon is quite distinct but Trimeresurus flavoviridis did not differ immunologically from the three species of Botbrops studied.

It would appear that the study of the shared antigens in snake venom is of taxonomic value both at the species group and the family levels. Occasionally, however, antigenic proteins in snakes venom are known to evolve rapidly, especially in isolated populations, and consequently the presence of a relatively small proportion of shared antigens should not necessarily be taken as evidence of a lack of affinity between two taxa.
\end{abstract}

\section{RESUME}

L'étude, par immunoélectrophorèse et par immunodiffusion, des communautés antigéniques des venins de 21 espèces de Viperidae, appartenant à 10 genres différents, apporte un certain nombre de données immunotaxonomiques, schématisées sur le dendrogramme de la fig. 1. La plupart de ces résultats confirment simplement la classification habituelle, basée sur la morphologie comparée et la biogéographie. D'autres, tels que la position un peu à part de Atheris, demandent à être confirmés sur un matériel plus important. Certains résultats, enfin, doivent être pris en considération lors d'une reconstitution phylogénique des Viperidae. Parmi les Viperinae, les genres Echis et Cerastes paraissent très proches l'un de l'autre et plus proches du genre Bitis que du genre Vipera. Dans ce dernier genre, on distingue clairement trois groupes d'espèces, Vipera russelli ayant une position assez isolée. Parmi les Crotalinae, le genre Agkistrodon se différencie nettement, tandis que Trimeresurus flavoviridis ne se distingue pas des trois espèces de Bothrops étudiées.

II semble bien que l'étude des communautés antigéniques des venins de Serpents présente un réel intérêt taxonomique à un niveau allant du groupe d'espèces à la famille. Cependant, les protéines antigéniques des venins peuvent évoluer très rapidement dans des populations isolées; en conséquence, une proportion relativement faible d'antigènes communs n'indique pas nécessairement l'absence d'affinités étroites entre deux taxons.

\section{INTRODUCTION}

En raison de la rareté des données paléontologiques, la systématique des Serpents est principalement basée sur la morphologie comparée des formes actuelles, dont l'interprétation est parfois délicate dans ce groupe de structures assez uniformes. En règle générale, les super-familles se différencient nettement, encore que la position respective des Typhlopoidea et des Leptotyphlopoidea prête toujours à discussion. Mais les taxons de niveau compris entre la famille et le genre sont souvent difficiles à caractériser, comme le montrent les fluctuations de la nomenclature. L'utilisation de critères biochimiques est donc tentante. Jusqu'à présent, les recherches de cet ordre ont surtout porté sur le sérum sanguin, soit par électrophorèse simple (Voris, 1967), soit par analyse immunologique (Domergue et al., 1969; Pearson, 1968). Au niveau taxonomique envisagé ici, c'est cette dernière méthode qui a apporté les résultats les plus intéressants.

Les protéines des venins ont fait l'objet de nombreuses recherches, mais leurs résultats n'ont guère été appliqués à la systématique des Serpents, 
sauf parfois à des niveaux infra-génériques (Schenberg, 1963; Tu \& Adams, 1968; Tu \& Ganthavorn, 1978; Saint Girons \& Detrait, 1978). Disposant d'assez nombreux lots de venins et des immunsérums monospécifiques correspondants, il nous a semblé intéressant d'utiliser ce matériel à une étude immuno-taxonomique des Viperidae.

Ce travail a été fait grâce à des crédits d'aide individuelle du C.N.R.S. Par ailleurs, nous tenons à remercier notre Collègue $\mathrm{D}$. Bradshaw qui a bien voulu se charger de la traduction en anglais du résumé.

\section{MATERIEL ET TECHNIQUES}

Pour cette étude, nous avons utilisé essentiellement 13 lots de venins et les immun-sérums homologues préparés à partir de ces mêmes lots. Ces venins correspondent à des pools très importants, recueillis sur de nombreux individus dans une aire géographique généralement étendue, mais parfois imprécise. Ils proviennent des espèces suivantes: Vipera berus berus (Russie), $V$. aspis aspis (France), $V$. ammodytes ammodytes (Yougoslavie), V. lebetina deserti (Tunisie), V. palestinae (Israël), V. russelli (Asie méridionale), Cerastes cerastes (Afrique), Ecbis carinatus pyramidum (Afrique), Bitis lachesis lachesis (Afrique occidentale), B. gabonica (Afrique occidentale), Agkistrodon rbodostoma (Cambodge), Bothrops lanceolatus (Martinique) et Crotalus durissus terrificus (Brésil). Desséchés sous vide et finement broyés, ces venins sont conservés à $+4^{\circ} \mathrm{C}$. Au moment de l'emploi, ils sont dissous dans du sérum physiologique à neuf pour mille, à raison de $10 \mathrm{mg} / \mathrm{ml}$.

Les immun-sérums, d'origine équine à l'exception des sérums anti-venins de $V$. berus et de $V$. lebetina qui sont d'origine caprine, proviennent tous d'animaux hyperimmunisés par injections de doses croissantes de venins et sont, bien entendu, monovalents. Ces immun-sérums ont tous été utilisés à l'état brut, c'est à dire non purifiés. Lyophilisés et conservés à $+4^{\circ} \mathrm{C}$ ils sont, pour les expériences, remis en solution dans de l'eau distillée à raison de $100 \mathrm{mg}$ de produit sec par $\mathrm{ml}$.

Nous avons, en outre, utilisé les venins de 8 autres espèces, pour lesquels nous ne possédions pas d'immun-sérum homologue. Il s'agit en général de lots beaucoup moins importants que les précédents, provenant de Bitis nasicornis (Afrique occidentale), Atheris sp. (Afrique occidentale), Causus rbombeatus (Afrique occidentale), Agkistrodon piscivorus (Etats Unis), Botbrops atrox atrox (Brésil), Botbrops jararaca (Brésil), Trimeresurus flavoviridis (Japon) et Crotalus adamanteus (Etats Unis).

L'analyse immunoélectrophorétique a été faite selon la technique précédemment décrite (Saint Girons \& Detrait, 1978). Pour les immunodiffusions, basées sur la technique d'Ouchterlony, nous avons également utilisé des plaques de verre de $13 \times 18 \mathrm{~cm}$, recouvertes d'un gel d'agarose (Indubiose A 37) à $2 \%$ en sérum physiologique, sur une épaisseur de $3 \mathrm{~mm}$. Des cupules d'un diamètre de $2,7 \mathrm{~mm}$ et des gouttières de $1,4 \mathrm{~mm}$ de largeur sont disposées comme on peut le voir sur la fig. 1 de la planche. Les solutions de venin sont distribuées à raison de $10 \mu \mathrm{l}$ dans chacune des cupules, le venin homologue étant toujours intercalé entre les différents venins hétérologues, puis les immun-sérums sont déposés sous un volume de $0,40 \mathrm{ml}$ dans les gouttières. Les plaques sont ensuite traitées comme les immunoélectrophorèses: repos de deux jours dans un incubateur à la température du laboratoire, lavage dans du sérum physiologique durant trois jours, séchage, coloration à la Rhodamine et différenciation.

Comme précédemment, le décompte des lignes de précipités est fait en plaçant les plaques dans une agrandisseur photographique et en dessinant les images obtenues, à un agrandissement de 3 à 4 diamètres. Nous avons utilisé le test $t$ de Student pour comparer les moyennes obtenues.

\section{RESULTATS}

Lorsque le venin d'une espèce est testé avec l'immun-sérum homologue, selon la méthode de l'immunodiffusion, 9 à 12 lignes de précipités apparaissent, regroupées en 4 à 6 systèmes de précipitation. Pour un même immun-sérum, il est souvent possible de reconnaître quelles sont, vis à vis des venins hétérologues, les lignes de précipités qui manquent. Toutefois, lorsque les communautés antigéniques sont faibles, les quelques lignes qui subsistent, peu nettes et généralement décalées vers la source des anticorps, ne peuvent plus être individualisées. Il est également impossible d'établir des homologies strictes entre les différents systèmes de précipitation d'un immun-sérum à l'autre. Pour ces raisons, ce travail est essentiellement basé sur le nombre des antigènes communs révélés par les réactions de précipitation, nombre qui figure sur le tableau I.

En immunoélectrophorèse, la migration des protéines dans le champ électrique permet de différencier un plus grand nombre de lignes de précipités, de 14 à 19 (le plus souvent de 16 à 18) pour des réactions homologues. Toutefois, plus encore qu'en immunodiffusion, il est impossible d'établir des homologies entre les différentes lignes de précipités, non seulement d'un immun-sérum à l'autre, mais également pour le même immun-sérum testé avec différents venins. Le nombre des antigènes communs révélés dans ces conditions figure sur le tableau II.

Comme le nombre absolu des lignes de précipités discernables lors des réactions homologues varie d'un immun-sérum à l'autre, il est plus logique de travailler sur la proportion des antigènes communs. Malgré cette rectification, on constate que les résultats obtenus en testant, d'une part le venin a avec l'immun-sérum $b$, d'autre part le venin $b$ 
TABleAu I

Immunodiffusions. Nombre de lignes de précipités discernables.

Immun-sérums

V.ber. V.asp. V.amm. V.leb. V.pal. V.rus C.cer. E.car. B.lac. B. gab. A. rbo. B.lan. C.dur.

\begin{tabular}{|c|c|c|c|c|c|c|c|c|c|c|c|c|c|}
\hline \multicolumn{14}{|l|}{ Venins } \\
\hline Vipera berus & 11 & 7 & 6 & 5 & 4 & 5 & 4 & 4 & 5 & 2 & 1 & 2 & 2 \\
\hline Vipera aspis & 6 & 11 & 6 & 5 & 4 & 5 & 4 & 4 & 6 & 3 & 1 & 2 & 2 \\
\hline Vipera ammodytes & 6 & 7 & 11 & 5 & 4 & 5 & 5 & 4 & 4 & 2 & 0 & 2 & 2 \\
\hline Vipera lebetina & 4 & 5 & 5 & 10 & 7 & 4 & 5 & 3 & 5 & 2 & 0 & 2 & 2 \\
\hline Vipera palestinae & 5 & 5 & 6 & 7 & 11 & 5 & 5 & 3 & 5 & 2 & 1 & 2 & 2 \\
\hline Vipera russelli & 3 & 4 & 5 & 4 & 4 & 11 & 4 & 3 & 5 & 3 & 1 & 3 & 1 \\
\hline Cerastes cerastes & 1 & 1 & 2 & 2 & 2 & 1 & 12 & 9 & 6 & 2 & 1 & 1 & 0 \\
\hline Echis carinatus & 2 & 2 & 2 & 3 & 2 & 1 & 9 & 12 & 6 & 3 & 1 & 2 & 1 \\
\hline Bitis lachesis & 2 & 2 & 3 & 2 & 2 & 2 & 6 & 4 & 11 & 5 & 1 & 1 & 3 \\
\hline Butis gabonica & 2 & 2 & 3 & 2 & 3 & 2 & 6 & 4 & 8 & 9 & 1 & 1 & 3 \\
\hline Bitis nasicornis & & 3 & 3 & 4 & & 5 & 3 & 3 & 7 & 6 & & & \\
\hline Atberis sp. & & 2 & 2 & 2 & & 2 & 2 & 3 & 2 & 3 & & & \\
\hline Causus rbombeatus & & 2 & 2 & 2 & & 1 & 1 & 2 & 2 & 2 & 0 & 0 & 0 \\
\hline Agkistrodon rbo. & 1 & 1 & 1 & 1 & 0 & 1 & 2 & 1 & 2 & 0 & 12 & 2 & 3 \\
\hline Agkistrodon pis. & & & & & & & & & & & 2 & 1 & 2 \\
\hline Bothrops lanceol. & 2 & 1 & 1 & 1 & 1 & 1 & 2 & 2 & 1 & 0 & 1 & 9 & 5 \\
\hline Bothrops atrox & & & & & & & & & & & 1 & 4 & 3 \\
\hline Bothrops jararaca & & & & & & & & & & & 0 & 3 & 3 \\
\hline Trimeresurus fla. & & & & & & & & & & & 0 & 2 & 2 \\
\hline Crotalus durissus & 2 & i & 1 & 1 & 1 & 2 & 2 & 3 & 2 & 0 & 0 & 4 & 10 \\
\hline Crotalus adamant. & & & & & & & & & & & 0 & 1 & 5 \\
\hline
\end{tabular}

TABLEAU II

Immunoélectrophorèses. Nombre de lignes de précipités discernables.

Immun-sérums

V.ber. V.asp. V.amm. V.leb. V. pal. V.rus C.cer. E.car, B.lac. B. gab. A.rbo. B.lan. C.dur.

\begin{tabular}{|c|c|c|c|c|c|c|c|c|c|c|c|c|c|}
\hline Venins & & & & & & & & & & & & & \\
\hline Vipera berus & 17 & 12 & 10 & 9 & 7 & 6 & 7 & 6 & 6 & 4 & 2 & 4 & 3 \\
\hline Vipera aspis & 11 & 17 & 10 & 8 & 7 & 7 & 9 & 8 & 7 & 5 & 2 & 3 & 3 \\
\hline Vipera ammodytes & 9 & 10 & 18 & 9 & 8 & 7 & 9 & 6 & 6 & 4 & 2 & 3 & 3 \\
\hline Vipera lebetina & 8 & 9 & 9 & 17 & 12 & 8 & 7 & 5 & 6 & 3 & 1 & 3 & 3 \\
\hline Vipera palestinae & 9 & 10 & 10 & 13 & 16 & 10 & 9 & 5 & 7 & 4 & 2 & 5 & 3 \\
\hline Vipera russelli & 7 & 8 & 7 & 8 & 7 & 18 & 7 & 5 & 7 & 4 & 2 & 2 & 3 \\
\hline Cerastes cerastes & 4 & 4 & 4 & 6 & 4 & 6 & 16 & 13 & 7 & 5 & 1 & 3 & 3 \\
\hline Echis carinatus & 5 & 4 & 3 & 5 & 3 & 4 & 12 & 17 & 7 & 5 & 1 & 3 & 2 \\
\hline Bitis lacbesis & 3 & 4 & 3 & 5 & 3 & 5 & 9 & 6 & 19 & 6 & 2 & 3 & 3 \\
\hline Bitis gabonica & 4 & 6 & 5 & 6 & 6 & 6 & 10 & 9 & 11 & 17 & 2 & 4 & 4 \\
\hline Bitis nasicornis & 6 & 6 & 5 & 6 & 5 & 6 & 9 & 8 & 13 & 12 & 1 & 3 & 3 \\
\hline Atheris sp. & 3 & 3 & 4 & 5 & 3 & 5 & 4 & 3 & 4 & 4 & 1 & 2 & 3 \\
\hline Causus rbombeatus & 2 & 2 & 2 & 3 & 2 & 3 & 3 & 3 & 4 & 2 & 0 & 2 & 2 \\
\hline Agkistrodon rbo. & 3 & 2 & 2 & 1 & 2 & 3 & 3 & 3 & 3 & 2 & 16 & 3 & 4 \\
\hline Agkistrodon pis. & 3 & 3 & 3 & 2 & 2 & 3 & 5 & 3 & 3 & 1 & 3 & 4 & 4 \\
\hline Botbrops lanceol. & 3 & 2 & 2 & 3 & 2 & 3 & 5 & 4 & 3 & 2 & 1 & 14 & 6 \\
\hline Bothrops atrox & 3 & 3 & 3 & 2 & 3 & 3 & 5 & 4 & 5 & 2 & 1 & 6 & 6 \\
\hline Botbrops jararaca & 3 & 3 & 2 & 2 & 1 & 3 & 5 & 4 & 4 & 3 & 0 & 6 & 6 \\
\hline Trimeresurus fla. & 5 & 4 & 5 & 3 & 4 & 5 & 6 & 5 & 5 & 5 & 4 & 7 & 5 \\
\hline Crotalus durissus & 3 & 2 & 1 & 2 & 1 & 3 & 5 & 4 & 3 & 2 & 1 & 5 & 16 \\
\hline Crotalus adamant. & 3 & 1 & 1 & 1 & 1 & 2 & 4 & 4 & 3 & 2 & 0 & 5 & 8 \\
\hline
\end{tabular}




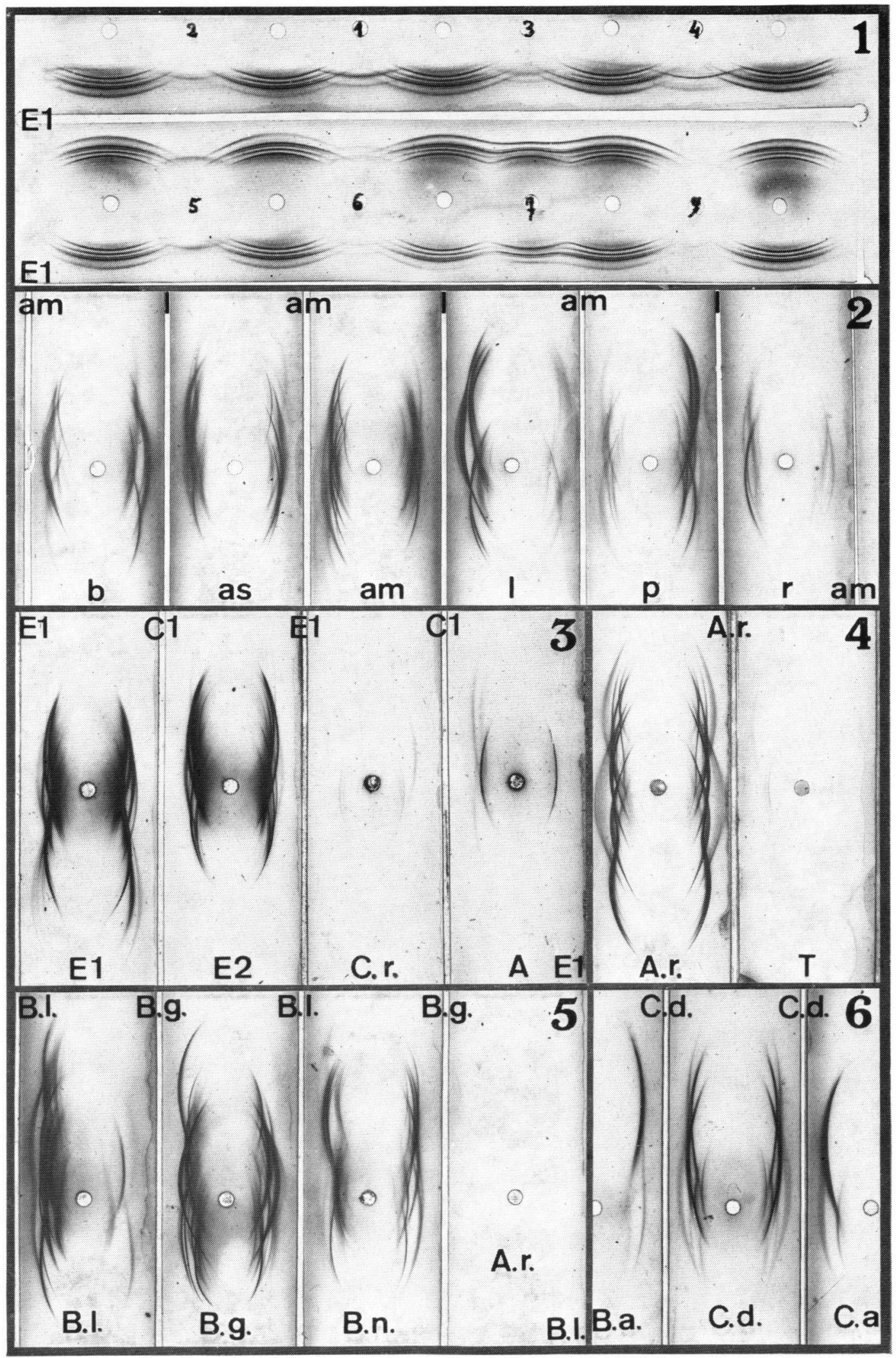


TABLEAU III

Pourcentage d'antigènes communs dans le venin de divers Viperidae.

Immunoélectrophorèses

V. ber. V.asp. V. amm. V.leb, V.pal. V.rus. C. cer. E. car. B. lac. B. gab. A. tho. B. lan. C.dur.

\begin{tabular}{|c|c|c|c|c|c|c|c|c|c|c|c|c|c|}
\hline Bitis nasicornis & 35.3 & 35.3 & 27.8 & 35.3 & 31.3 & 33.3 & 56.3 & 47.1 & 68.4 & 70.6 & 6.3 & 21.4 & 18.8 \\
\hline Atheris sp. & 17.6 & 17.6 & 22.2 & 29.4 & 18.6 & 27.8 & 25.0 & 17.6 & 21.1 & 23.5 & 6.3 & 14.3 & 18.8 \\
\hline Causus rhombeat. & 11.8 & 11.8 & 11.1 & 17.6 & 12.5 & 16.7 & 18.8 & 17.6 & 21.1 & 11.8 & 0.0 & 14.3 & 12.5 \\
\hline Agkistrodon pis. & 17.6 & 17.6 & 16.7 & 11.8 & 12.5 & 16.7 & 31.3 & 17.6 & 15.8 & 5.9 & 18.8 & 28.6 & 25.0 \\
\hline Botbrops atrox & 17.6 & 17.6 & 16.7 & 11.8 & 18.8 & 16.7 & 31.3 & 23.5 & 26.3 & 11.8 & 6.3 & 42.9 & 37.5 \\
\hline Botbrops jararaca & 17.6 & 17.6 & 11.1 & 11.8 & 6.3 & 16.7 & 31.3 & 23.5 & 21.1 & 17.6 & 0.0 & 42.9 & 37.5 \\
\hline Trimeresurus fla. & 29.4 & 23.5 & 27.8 & 17.6 & 25.0 & 27.8 & 37.5 & 29.4 & 26.3 & 29.4 & 25.0 & 50.0 & 31.3 \\
\hline Crotalus adamant. & 17.6 & 5.9 & 5.6 & 5.9 & 6.3 & 11.1 & 25.0 & 23.5 & 15.8 & 11.8 & 0.0 & 35.7 & 50.0 \\
\hline Vipera berus & & 67.7 & 54.3 & 50.0 & 48.4 & 37.3 & 33.7 & 32.4 & 24.6 & 23.5 & 15.1 & 23.1 & 18.2 \\
\hline Vipera aspis & 59.1 & & 57.2 & 50.0 & 51.3 & 43.0 & 39.9 & 35.3 & 30.2 & 32.4 & 12.2 & 16.6 & 15.3 \\
\hline Vipera ammodytes & 54.5 & 59.1 & & 51.5 & 52.8 & 38.9 & 39.3 & 26.0 & 24.2 & 25.7 & 14.6 & 16.3 & 12.2 \\
\hline Vipera lebetina & 45.0 & 47.8 & 47.8 & & 75.8 & 45.8 & 39.6 & 32.4 & 30.5 & 26.5 & 6.1 & 19.5 & 15.3 \\
\hline Vipera palestinae & 43.2 & 41.0 & 45.5 & 66.7 & & 49.7 & 40.7 & 27.1 & 27.8 & 30.4 & 12.5 & 24.1 & 12.6 \\
\hline Vipera russelli & 36.4 & 41.0 & 45.5 & 38.2 & 41.0 & & 38.6 & 28.8 & 32.3 & 28.4 & 14.6 & 15.5 & 17.8 \\
\hline Cerastes cerast. & 21.2 & 21.2 & 30.0 & 30.9 & 30.0 & 21.2 & & 75.8 & 46.6 & 46.0 & 12.6 & 26.4 & 25.1 \\
\hline Echis carinatus & 25.8 & 25.8 & 25.8 & 27.5 & 21.6 & 17.1 & 75.0 & & 36.1 & 41.2 & 12.0 & 22.5 & 18.0 \\
\hline Bitis lachesis & 31.9 & 36.4 & 31.9 & 32.8 & 31.9 & 31.9 & 52.3 & 43.9 & & 46.6 & 14.2 & 18.6 & 17.3 \\
\hline Bitis gabonica & 20.2 & 24.3 & 24.8 & 21.9 & 24.8 & 25.8 & 36.1 & 33.3 & 64.2 & & 9.2 & 20.2 & 18.4 \\
\hline Agkistrodon rbo. & 9.1 & 9.1 & 4.6 & 5.0 & 4.6 & 9.1 & 12.9 & 8.7 & 13.7 & 4.6 & & 13.9 & 15.7 \\
\hline Botbrops lanceol. & 20.2 & 15.7 & 15.7 & 16.1 & 15.7 & 25.8 & 13.9 & 19.5 & 10.1 & 5.6 & 17.5 & & 36.6 \\
\hline Crotalus durissus & 19.1 & 14.6 & 14.6 & 15.0 & 14.6 & 14.1 & 8.4 & 17.5 & 24.1 & 15.0 & 15.0 & 47.2 & \\
\hline Bitis nasicornis & & 27.3 & 27.3 & 40.0 & & 45.5 & 25.0 & 25.0 & 54.5 & 66.7 & & & \\
\hline Atheris sp. & & 18.2 & 18.2 & 20.0 & & 18.5 & 16.7 & 25.0 & 18.2 & 33.3 & & & \\
\hline Causus rbombeatus & & 18.2 & 18.2 & 20.0 & & 9.1 & 8.3 & 16.7 & 18.2 & 22.2 & & & \\
\hline Agkistrodon pis. & & & & & & & & & & & 16.7 & 11.1 & 20.0 \\
\hline Botbrops atrox & & & & & & & & & & & 8.3 & 44.4 & 30.0 \\
\hline Botbrops jararaca & & & & & & & & & & & 0.0 & 33.3 & 30.0 \\
\hline Trimeresurus fla. & & & & & & & & & & & 0.0 & 22.2 & 20.0 \\
\hline Crotalus adamant. & & & & & & & & & & & 0.0 & 11.1 & 50.0 \\
\hline & \multicolumn{13}{|c|}{ Immunodiffusions } \\
\hline
\end{tabular}

Planche I. - Communautés antigéniques des venins chez divers Viperidae.

1. Immunodiffusion. Immun-sérum de Echis carinatus (E1). Venins de Vipera aspis (2), $V$. berus (1), V. ammodytes (3), $V$. lebetina (4), V. palestinae (5), V. russelli (6), Cerastes cerastes (7) et Bitis lachesis (9). Le venin homologue de Echis carinatus (puits sans numéro) alterne avec celui des autres espèces.

2-6. Immunoélectrophorèses.

2. Immun-sérums de Vipera ammodytes (am) et de $V$. lebetina (1). Venins de Vipera berus (b), V. aspis (as), V. ammodytes (am), $V$. lebetina (1), $V$. palestinae $(\mathrm{p})$ et $V$, russelli $(\mathrm{r})$.

3. Immun-sérums de Echis carinatus (E1) et de Cerastes cerastes (C1). Venins de Echis carinatus (E1 et E2, explications sur le tableau IV), Causus rhombeatus (C.r.) et Atberis sp. (A).

4. Immun-sérum de Agkistrodon rbodostoma (A.r.). Venins de Agkistrodon rbodostoma (A.r.) et Trimeresurus flavoviridis $(\mathrm{T})$.

5. Immun-sérums de Bitis lachesis (B.1.) et de B. gabonica (B.g.). Venins de Bitis lachesis (B.I.), B. gabonica (B.g.), B. nasicornis (B.n.) et Agkistrodon rhodostoma (A.r.).

6. Immun-sérum de Crotalus durissus (C.d.). Venin de Bothrops atrox (B.a.), Crotalus durissus (C.d.) et C. adamanteus (C.a.).

Sauf pour les réactions homologues faites isolément (ici Agkistrodon rbodostoma), la différenciation a été volontairement peu poussée, afin de ne pas perdre les lignes de précipités faibles, ce qui explique partiellement la mauvaise qualité des clichés. 
TABLEAU IV

Communautés antigéniques entre divers lots de venins de Echis carinatus et de Cerastes cerastes.

\begin{tabular}{|c|c|c|c|c|c|c|c|c|c|c|}
\hline \multirow[b]{2}{*}{ Venins } & \multicolumn{6}{|c|}{$\begin{array}{l}\text { Immunodiffusions } \\
\text { Immun-sérums }\end{array}$} & \multicolumn{4}{|c|}{$\begin{array}{l}\text { Immunoélectrophorèses } \\
\text { Immun-sérums }\end{array}$} \\
\hline & E1 & E2 & $\mathrm{C} 1$ & E1 & E2 & $\mathrm{C} 1$ & $\mathrm{E}_{1}$ & $\mathrm{C}_{1}$ & E1 & $\mathrm{Cl}$ \\
\hline Echis (Ethiopie) E1 & 12 & 8 & 9 & 100.0 & 80.0 & 75.0 & 17 & 12 & 100.0 & 75.0 \\
\hline Echis (Afrique) E2 & 10 & 10 & 8 & 83.3 & 100.0 & 66.7 & 13 & 11 & 76.5 & 68.8 \\
\hline Echis (Pakistan) & 7 & & 8 & 58.3 & & 66.7 & 9 & 9 & 52.9 & 56.3 \\
\hline Cerastes (Ethiopie) $\mathrm{C}_{1}$ & 9 & 7 & 12 & 75.0 & 70.0 & 100.0 & 13 & 16 & 76.5 & 100.0 \\
\hline \multirow[t]{2}{*}{ Cerastes (Tunisie) } & 7 & 7 & 8 & 58.3 & 70.0 & 66.7 & 7 & 11 & 41.2 & 68.8 \\
\hline & \multicolumn{3}{|c|}{ Nombres } & \multicolumn{3}{|c|}{ Pourcentages } & \multicolumn{2}{|c|}{ Nombres } & \multicolumn{2}{|c|}{ Pourcentages } \\
\hline
\end{tabular}

avec l'immun-sérum a, sont généralement voisins lorsque la proportion des antigènes communs est élevée, mais varient parfois du simple au double dans le cas contraire. Pour simplifier, nous avons pris la moyenne entre chacun de ces couples de données (tableau III), étant entendu que les chiffres obtenus lorsque les communautés antigéniques sont faibles n'indiquent plus qu'un ordre de grandeur. A ces 13 réactions croisées, nous avons joint l'analyse des venins des 8 espèces pour lesquelles nous ne possédions pas d'immun-sérum homologue. Il va de soi que ces résultats ne sont pas tout à fait comparables aux précédents, mais il est néanmoins possible d'en tenir compte.

Dans l'ensemble, la corrélation du nombre de lignes de précipités obtenues par immunodiffusion et par immunoélectrophorèse est assez bonne, le meilleur ajustement correspondant à une régression linéaire $(r=0,96, a=0,74, b=1,45$, $p<0,001$ pour 161 couples de données). Toutefois, assez curieusement, il n'existe pas de corrélation significative dans le cas des réactions homologues considérées seules $(r=0,35, p>0,10)$.

Il ressort du tableau III que Viperinae et Crotalinae, bien que certainement apparentés, n'ont que peu d'antigènes communs dans leurs venins: $16,17 \pm 0,83$ ou $13,22 \pm 1,02 \% 1$. Il en est d'ailleurs de même entre Causus rbombeatus et les Viperinae $(15,08 \pm 1,16$ ou $16,36 \pm 1,77 \%)$. Parmi ces derniers, l'unique lot de venin de Atheris

1 Dans le texte, nous donnons la moyenne et l'erreur standard, successivement pour les résultats de l'immunoélectrophorèse, puis de l'immunodiffusion. Le nombre des données peut être facilement retrouvé sur le tableau III. sp. se différencie nettement, puisque l'on ne trouve que $22,04 \pm 1,37$ ou $21,01 \pm 1,97 \%$ d'antigènes communs avec les autres espèces. Le genre Vipera a déjà $31,62 \pm 0,91$ ou $27,71 \pm 1,20 \%$ d'antigènes communs avec les trois autres genres, tandis que, parmi ces derniers, Cerastes et Echis, extrêmement proches l'un de l'autre ( 75 à $75,8 \%$ d'antigènes communs), sont apparemment plus proches de Bitis (en moyenne 45,55 $\pm 2,75$ ou 35,13 \pm $4,39 \%$ d'antigènes communs) que de Vipera $(34,48 \pm 1,52$ ou $24,84 \pm 1,26 \%)$, la différence étant significative avec les deux méthodes $(p<0,05$ pour Cerastes, $<0,02$ pour Echis et $<0,01$ pour l'ensemble).

La proportion très élevée d'antigènes communs entre Cerastes cerastes et Echis carinatus est inattendue. Comme les deux lots de venins provenaient de la même source commerciale, en Ethiopie, une contamination pouvait être envisagée (voir à ce sujet Boquet et al., 1969). Aussi avons-nous refait les expériences avec un autre lot de venin d'Echis carinatus d'Afrique et son immun-sérum homologue, avec un venin d'Echis carinatus du Pakistan et avec un venin de Cerastes cerastes provenant de Tunisie (tableau IV). L'hypothèse d'une contamination n'est pas exclue, puisque la proportion d'antigènes communs entre les deux lots hétérologues d'Ethiopie est la plus élevée, mais nous trouvons encore $70 \%$ d'antigènes communs entre les autres Echis d'Afrique et Cerastes de Tunisie, c'est à dire plus qu'entre Echis d'Afrique et du Pakistan, les chiffres les plus bas étant relevés entre Echis d'Ethiopie et Cerastes de Tunisie.

Les rapports des différentes Bitis avec les représentants des autres genres sont assez variables. En 
effet, l'immun-sérum de $B$. gabonica donne nettement moins de lignes de précipités avec les venins des autres Viperinae que l'immun-sérum de $B$. lachesis $(24,09 \pm 1,38$ ou $26,36 \pm 2,03 \%$, au lieu de $34,72 \pm 0,85$ ou $47,74 \pm 2,26 \%$, $p<0,001$ avec les deux méthodes), ce qui n'est pas le cas lorsque l'on teste les venins de ces deux espèces avec les immun-sérums des autres Viperinae. Néanmoins, chaque espèce de Bitis est plus proche de Cerastes et de Echis que de Vipera, la seule exception étant représentée par $B$. nasicornis en immunodiffusion, mais non en immunoélectrophorèse.

A l'intérieur du genre Vipera on distingue nettement trois groupes d'espèces: tout d'abord $V$. russelli qui n'a en commun que 42,94 $\pm 2,26$ ou $40,42 \pm 1,54 \%$ d'antigènes communs avec les autres espèces, ensuite $V$. lebetina et $V$. palestinae qui ont 75,8 ou $66,7 \%$ d'antigènes communs entre elles, mais seulement $50,67 \pm 0,62$ ou 45,05 \pm $1,08 \%$ avec le troisième groupe constitué par les petites Vipères d'Eurasie tempérée et froide. Parmi ces dernières, en immunoélectrophorèse $V$. aspis et $V$. berus sont plus proches l'une de l'autre $(67,7 \%$ d'antigènes communs) que de $V$. ammodytes $(54,3$ et $57,2 \%)$, alors qu'en immunodiffusion les trois espèces sont à peu près à égale distance les unes des autres $(54,5$ à $59,1 \%)$. Les rapports entre les trois espèces de Bitis sont d'interprétation moins évidente. Dans l'ensemble, la proportion des antigènes communs est élevée et voisine de $62 \%$ en moyenne, avec les deux méthodes, $B$. nasicornis et $B$. gabonica étant plus proches l'une de l'autre $(70,6$ ou $66,7 \%$ d'antigènes communs) que de $B$. lachesis $(57,5$ ou $59,3 \%)$.

Les données concernant les Crotalinae sont moins nombreuses. Apparemment, les Ancistrodons diffèrent nettement des autres genres, avec lesquels ils n'ont, en moyenne, que $14,31 \pm 4,03$ ou $8,99 \pm$ $2,92 \%$ d'antigènes communs. Il convient toutefois de noter que l'immun-sérum de Agkistrodon rbodostoma réagit particulièrement peu avec les différents venins des autres Crotalinae (7,32 $\pm 3,75$ ou $2,9 \pm 1,84 \%$ d'antigènes communs), alors que le venin de cette espèce et celui de $A$. piscivorus donnent beaucoup plus de lignes de précipités avec les immun-sérums de Bothrops lanceolatus et de Crotalus durissus ( $25 \pm 1,47$ ou $20,85 \pm 3,87 \%$ ).
Vis à vis des Viperinae, toutes ces réactions sont également faibles.

Les autres Crotalinae se répartissent en deux groupes, Bothrops et Trimeresurus d'une part, Crotalus de l'autre, qui ont en moyenne 35,72 $\pm 1,15$ ou $27,66 \pm 6,02 \%$ d'antigènes communs entre eux. Trimeresurus flavoviridis ne diffère pas plus des trois Botbrops que ceux-ci ne diffèrent entre eux (en moyenne $45,27 \pm 2,37$ ou $33,3 \pm 6,41 \%$ d'atigènes communs), les deux Crotalus ayant, par les deux méthodes, $50 \%$ d'antigènes communs. $\mathrm{Au}$ contraire, les communautés antigèniques entre Agkistrodon rbodostoma et $A$. piscivorus sont faibles: 18,8 ou $16,7 \%$.

\section{DISCUSSION}

Une discussion détaillée des méthodes utilisées et de leurs limites sortirait du cadre de cet article (voir à ce sujet Grasset et al., 1956 et Jouannet, 1968). Rappelons seulement que les paramètres impliqués sont si nombreux que, même avec des techniques aussi standardisées que possible, les résultats ne sont pas toujours strictement reproductibles. D'autre part, ce travail est basé sur le nombre des lignes de précipités discernables, sans tenir compte de leur intensité, et des lignes tout juste perceptibles, décalées vers la source des anticorps et correspondant vraisemblablement à des réactions paraspécifiques, sont néanmoins comptées comme des unités. Les chiffres qui figurent sur les tableaux ont donc tendance à minimiser les différences, surtout lorsqu'il s'agit de communautés antigéniques faibles. Par ailleurs, l'étude de différents taxons d'un groupe d'espèces (Saint Girons \& Detrait, 1978) a montré que les protéines antigéniques des venins pouvaient, le cas échéant, évoluer très rapidement. En conséquence, si une proportion élevée d'antigènes communs suggère fortement des affinités phylogéniques étroites, l'inverse n'est pas obligatoirement vrai et des taxons apparentés peuvent avoir des venins assez différents.

La fig. 1 résume et schématise les communautés antigéniques des venins entre les différentes espèces étudiées, d'après les données de l'immunoélectrophorèse. Le dendrogramme obtenu d'après les résultats de l'immunodiffusion n'en diffère que par des détails mineurs. 


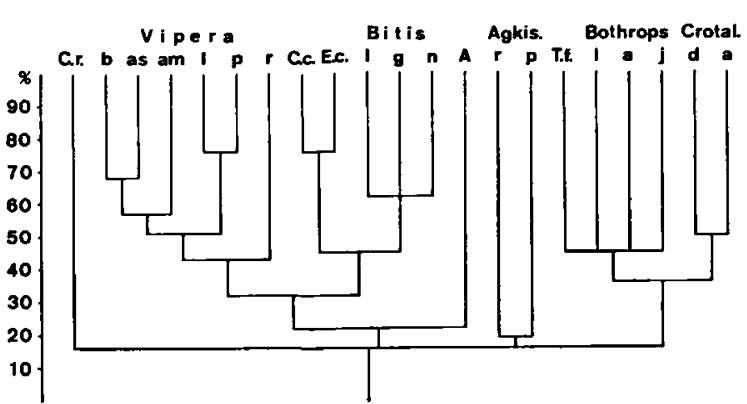

Fig. 1. Dendrogramme schématisant les communautés antigéniques de divers Viperidae, d'après le pourcentage moyen des antigènes communs dans les venins.

De gauche à droite: Causus rhombeatus (C.r.). Vipera: berus (b), aspis (as), ammodytes (am), lebetina (1), palestinae ( $\mathrm{p})$, russelli ( $\mathrm{r}$ ). Cerastes cerastes (C.c.), Echis carinatus (E.c.). Bitis: lacbesis (1), gabonica (g), nasicornis (n). Atberis sp. (A). Agkistrodon (Agkis.): rbodostoma ( $\mathrm{r}$ ), piscivorus (p). Trimeresurus flavoviridis (T.f.). Botbrops: lanceolatus (1), atrox (a), jararaca (j). Crotalus (Crotal.): durissus (d), adamanteus (a).

Parmi les Viperinae, la position très à part de Causus rhombeatus n'a rien d'étonnant puisque ce genre, bien que moins archaïque que Azemiops dont on a fait une sous-famille particulière (Liem et al., 1971), diffère nettement à de nombreux égards des autres Vipères. La faible proportion d'antigènes communs entre Atheris et les quatre autres genres de Viperinae $(22 \%)$ est plus inattendue et pourrait suggérer que cette lignée n'est pas aussi proche de celle des Bitis qu'on le pense généralement. Mais nous avons vu que des résultats négatifs n'ont pas obligatoirement de signification phylogénique, surtout lorsque le matériel étudié se limite à un seul petit lot de venin, ce qui est le cas ici. Il n'en est pas de même en ce qui concerne Cerastes et Echis qui présentent une proportion très élevée d'antigènes communs, alors que leurs ressemblances morphologiques pouvaient passer pour un phénomène de convergence dû à un mode de vie voisin. Cependant, les communautés antigéniques démontrent clairement une étroite parenté entre ces deux genres qui, en outre, sont nettement plus proches de Bitis que de Vipera. C'est là un résultat qui ne correspond pas aux conclusions de l'étude de Marx \& Rabb (1965), puisque ces auteurs rangent Cerastes et Echis dans le stock paléarctique, avec Vipera et Eristicophis et non dans le stock éthiopien qui comprend les genres Bitis, Atheris et Adenorbinos. En réalité, les don- nées biogéographiques ne contredisent nullement l'hypothèse selon laquelle Cerastes et Echis correspondraient à une spécialisation désertique de Vipères éthiopiennes. En effet, ces deux genres se retrouvent au sud du Sahara, Echis carinatus étant même largement répandue dans la zone soudanienne et, au contraire, rare dans la majeure partie de la Berbérie. Rappelons également que Bitis lachesis subsiste toujours dans le sud marocain, jusqu'à Agadir.

Notre échantillon de Bitis est trop restreint pour apporter des précisions sur les rapports des différentes espèces entre elles. Tout au plus pouvons-nous noter une plus grande proximité de $B$. nasicornis et de $B$. gabonica, deux espèces forestières, par rapport à $B$. lachesis, forme de savanes occupant même la zone sahélienne. En revanche, les communautés antigéniques des venins permettent de reconnaître trois groupes d'espèces dans le genre Vipera. Le premier est constitué par la seule Vipère de Russell, d'Asie méridionale et sudorientale, les deux suivants, plus proches l'un de l'autre, par les „lébétines" des régions méditerranéennes plus ou moins arides et par les „petites” Vipères d'Eurasie tempérée et froide. Ces résultats concordent étroitement avec les conclusions tirées des études morphologiques classiques (Boulenger, 1896; Saint Girons, 1978). V. russelli a d'ailleurs souvent été rangée dans un genre à part, Daboia.

B. Groombridge étudie actuellement la phylogénie des Viperinae à l'aide d'une analyse cladistique de nombreux caractères morphologiques et il sera très intéressant de comparer ses résultats avec ceux fournis par les communautés antigéniques des venins. D'après les données préliminaires qu'il a bien voulu nous communiquer, il semble qu'il y ait un bon accord, notamment en ce qui concerne la position très particulière de Causus, la proximité de Cerastes et Echis, leurs rapports plus étroits avec Bitis qu'avec Vipera et la position respective des différentes espèces du genre Vipera.

Les résultats concernant les Crotalinae sont beaucoup moins nets, ne serait-ce que parce que nous ne disposions que de trois immun-sérums monospécifiques. Le genre Agkistrodon diffère manifestement des autres représentants de la sousfamille, ce que la morphologie comparée et la biogéographie laissaient prévoir. Le très petit nom- 
bre d'antigènes communs entre les deux espèces étudiées ici est plus inattendu, mais correspond aux résultats obtenus par Tu et al. $(1968,1978)$. Ces auteurs ont étudié, par les mêmes méthodes, les venins de diverses espèces $d$ 'Ancistrodons, par rapport à deux immun-sérums de référence faits à partir des venins de $A$. rbodostoma de Thailand et de $A$. contortrix des Etats Unis. Apparemment, les communautés antigéniques sont d'autant plus faibles que les espèces sont plus éloignées géographiquement. Les auteurs ne donnent pas le nombre des lignes de précipités obtenues, mais d'après leurs figures on constate qu'il existe des communautés antigéniques très étroites (peut-être de l'ordre de 80 à $90 \%$ ) entre les différentes populations et sous-espèces, ainsi qu'entre $A$. contortrix et $A$. piscivorus. D'autre part, $A$. balys du Japon a sans doute 40 à $50 \%$ d'antigènes communs tant avec $A$. contortrix qu'avec $A$. rhodostoma. Vis à vis de cette dernière espèce, les communautés antigéniques sont de l'ordre de 20 à $30 \%$ avec $A$. contortrix, moins avec $A$. bilineata, moins encore avec $A$. piscivorus.

Pour beaucoup d'auteurs, les Trimeresurus ne sont que des Bothrops asiatiques et, effectivement, $T$. flavoviridis a autant d'antigènes communs avec $B$. lanceolatus que celui-ci avec $B$. atrox ou $B$. jararaca. La position systématique de Botbrops lanceolatus prête toutefois à discussion. D'aucun ne lui accordent aucun statut taxonomique (par exemple Klemmer, 1963), ou le considèrent comme une simple sous-espèce de $B$. atrox, alors que d'autres, notamment Hoge \& Romano (1971) estiment qu'il s'agit d'une espèce particulière. De toute façon, en raison de son isolement géographique, une évolution rapide des protéines du venin est très possible et ce phénomène pourrait expliquer le nombre relativement faible de lignes de précipités qui apparaissent lorsque l'on fait réagir l'immun-sérum de cette espèce avec les venins des autres Botbrops, y compris celui de $B$. atrox, forme au moins voisine. Les communautés antigéniques entre Trimeresurus et Botbrops d'une part, Crotalus de l'autre, sont de l'ordre de grandeur que l'on pouvait attendre d'après leur position systématique respective. Crotalus durissus et $C$. adamanteus, avec $50 \%$ d'antigènes communs, n'apparaissent pas particulièrement proches l'un de l'autre, ce qui est en bon accord avec la phylogénie proposée par Klauber (1956) d'après un ensemble de caractères morphologiques, éco-éthologiques et biogéographiques.

Parmi les nombreuses recherches faites sur les protéines sériques des Serpents, deux seulement portent sur les Viperidae, au niveau taxonomique envisagé ici. Le travail de Voris (1967) est basé sur un matériel très voisin du nôtre. Malheureusement, les résultats de ses électrophorèses sériques sont difficilement interprétables et l'auteur conclut lui-même ,this type of electrophoretic information is judged to have limited taxonomic use in this group". Il n'en est pas de même des données im. munologiques rassemblées par Pearson (1968) dans une étude des différentes familles de Serpents. D'après les résultats des immunoélectrophorèses sériques, il existe environ $57 \%$ d'antigènes communs entre les trois espèces de Crotalus testées et $26 \%$ entre Bitis gabonica et 5 Crotalinae. Dans l'ensemble, les communautés antigéniques paraissent un peu plus élevées dans le sérum sanguin que dans les venins et, a priori, leur signification phylogénique devrait être plus sûre. Toutefois, la grande variabilité du nombre des lignes de précipités obtenues lors des réactions homologues (de 5 à 16), introduit un facteur d'incertitude non négligeable.

\section{BIBLIOGRAPHIE}

Boquet, P., J. Detrait \& R. Farzampay, 1969. Recherches biochimiques et immunologiques sur le venin des Serpents. Ann. Inst. Pasteur, 116: 522-542.

Boulenger, G. A., 1896. Catalogue of snakes in the British Museum (Natural History), 3: 1-727. (Quaritch, London).

Domergue, C. A., A. Dodin, J. M. Pinon \& E. R. Brygoo, 1969. Première application des techniques sérologiques à l'étude de la systématique des Serpents de Madagascar. Arch. Inst. Pasteur Madagascar, 38: 175-180.

Grasset, E., E. Pongratz \& T. Brechbuhler, 1956. Analyse immunochimique des constituants des venins de Serpents par la méthode de précipitation en milieu gélifié. Ann. Inst. Pasteur, 91: 162-186.

Hoge, A. R. \& S. A. R. W. D. L. Romano, 1971. Neotropical pit vipers, sea snakes and coral snakes. In: W. BÜCHERL \& E. E. BuCKLEY, Venomous animals and their venoms, 2: 211-293. (Academic Press, New York, London).

JouANNET, M., 1968. L'analyse immuno-électrophorétique appliquée aux venins de Serpents. Toxicon, 5: 191-199.

KLAuber, L. M., 1956. Rattlesnakes, their habits, life histories, and influence on mankind, 2 vols.: 1-1476 (Univ. Calif. Press., Berkeley \& Los Angeles).

KLeMMER, K., 1963. Liste der rezenten Giftschlangen. In: Die Giftschlangen der Erde: 265-464. (N.G. Elwert Universitäts-Verlag, Marburg/Lahn). 
LIEM, K. F., H. MarX \& G. B. RABb, 1971. The viperid snake Azemiops: its comparative cephalic anatomy and phylogenetic position in relation to Viperinae and Crotalinae. Fieldiana (Zool.), 59: 65-126.

MARX, H. \& G. B. RABB, 1965. Relationship and zoogeography of the viperine snakes (family Viperidae). Fieldiana (Zool.), 44: 161-206.

PenRson, D. D., 1968. Immuno-taxonomic relationship among families of snakes. Proc. Pa. Acad. Sci., 42: 49-56.

SAINT Girons, H., 1978. Morphologie comparée et systématique des Vipères d'Europe. Rev. suisse Zool., 85: 565 595.

Saint Girons, H. \& J. Detrait, 1978. Communautés antigéniques des venins et systématique des Vipères euro- péennes. Etude immunoélectrophorétique. Bull. Soc. zool. Fr., 103: 155-166.

SCHEnberg, S., 1963. Immunological (Ouchterlony method) identification of intra subspecies qualitative differences in snakes venom composition. Toxicon, 1: 67-75.

Tu, A. T. \& B. L. ADAMS, 1968. Phylogenetic relationship among venomous snakes of the genus Agkistrodon from Asia and the North American continent. Nature (London), 217: 760-762.

Tu, A. T. \& S. Ganthavorn, 1978. Comparison of snake venoms (Reptilia, Serpentes) from Java, Indonesia and Thailand and its significance in evolution and biogeography. J. Herpetol., 12: 105-107.

VoRIs, H. K., 1967. Electrophoretic patterns of plasma proteins in the viperine snakes. Physiol. Zool., 40: 238-247.

Reçu: le 15 février 1979 